\title{
TITLE:
}

\section{Analysis of helical quadrupole focusing channel}

AUTHOR(S):

Morita, A; Iwashita, Y

\section{CITATION:}

Morita, A ... [et al]. Analysis of helical quadrupole focusing channel.

PHYSICAL REVIEW SPECIAL TOPICS-ACCELERATORS AND BEAMS 2003, 6(1): 014001.

ISSUE DATE:

2003-01

URL:

http://hdl.handle.net/2433/50334

RIGHT:

Copyright 2003 American Physical Society 


\title{
Analysis of helical quadrupole focusing channel
}

\author{
A. Morita and Y. Iwashita \\ ${ }^{1}$ Accelerator Laboratory, Nuclear Science Research Facility, Institute for Chemical Research, Kyoto University, \\ Gokanosho, Uji, Kyoto 611, Japan
}

(Received 19 July 2001; revised manuscript received 4 December 2002; published 8 January 2003)

A helical quadrupole focusing channel has continuous field symmetry and a stronger focusing power compared with a conventional FODO focusing channel. The good field symmetry allowed us to construct an explicit transfer matrix under the paraxial approximation. In the present paper, we report the paraxial analysis of the helical quadrupole focusing channel and compare its characteristics with those of a conventional FODO focusing channel.

DOI: 10.1103/PhysRevSTAB.6.014001

PACS numbers: 41.85.-p

\section{INTRODUCTION}

A helical quadrupole focusing channel (HQFC), a variation of a quadrupole focusing channel, consists of a long quadrupole field continuously twisted along the beam axis. Because of the dense population of the focusing element, the focusing power of a HQFC is stronger compared with a conventional FODO channel. The electrostatic version of a HQFC has been analyzed $[1,2]$ and tested [3-5]. The magnetic version of a HQFC has been treated as a series of rotating quadrupole slices [6] and analyzed using the Hamiltonian form [7]. However, analysis as beam optics was not achieved by LeCouteur [7]. Accurate analysis requires treatment of a field distribution that satisfies the Maxwell equation. Nonlinear effects are also discussed here using numerical simulations. This means that the model of the rotating quadrupole slices is just a linear approximation of the helical quadrupole, and is valid only around the axis; the field deviates from the quadrupole distribution with increase of the radius. A valid range of the model and an analytic solution with a 4D linear transfer matrix for a HQFC under paraxial approximation are discussed in the following sections. Simulation results including nonlinear effects are also shown in a later section.

\section{HELICAL QUADRUPOLE FIELD}

While the following discussions can be applied to both magnetic and electric helical quadrupoles, we will use notations for magnetic helical quadrupoles. In a free space, the helical quadrupole field satisfying the Maxwell equation is expressed by the following form using a modified Bessel function:

$$
\begin{gathered}
\Phi(r, \theta, z)=\frac{\Phi_{0}}{k} I_{2}(k r) \sin (2 \theta-k z) \\
\text { and } \quad \mathbf{B}=-\nabla \Phi,
\end{gathered}
$$

where $k$ is $2 \pi / L$ and $L$ is the period of the magnetic field (the period of a pole is $2 L$ ). Each field component in the cylindrical coordinate system is given as follows:

$$
\begin{gathered}
B_{r}(r, \theta, z)=-\Phi_{0} I_{2}^{\prime}(k r) \sin (2 \theta-k z), \\
B_{\theta}(r, \theta, z)=-2 \Phi_{0} \frac{I_{2}(k r)}{k r} \cos (2 \theta-k z), \\
\text { and } B_{z}(r, \theta, z)=\Phi_{0} I_{2}(k r) \cos (2 \theta-k z) .
\end{gathered}
$$

Figure 1 shows the $r$ dependences of the three components of the helical quadrupole field together with the $B_{r}$ component of the pure quadrupole. Around the center axis, the helical quadrupole field can be approximated by a rotating quadrupole with an angle of $\frac{k z}{2}$ rad. This paraxial approximation corresponds to the linear approximation. In order to examine the valid range of the linear approximation, we obtained the following expansions by Taylor series:

$$
\begin{aligned}
& B_{r}=-\frac{\Phi_{0}}{4}\left(k r+\frac{(k r)^{3}}{6}+O\left(r^{5}\right)\right) \sin (2 \theta-k z), \\
& B_{\theta}=-\frac{\Phi_{0}}{4}\left(k r+\frac{(k r)^{3}}{12}+O\left(r^{5}\right)\right) \cos (2 \theta-k z),
\end{aligned}
$$

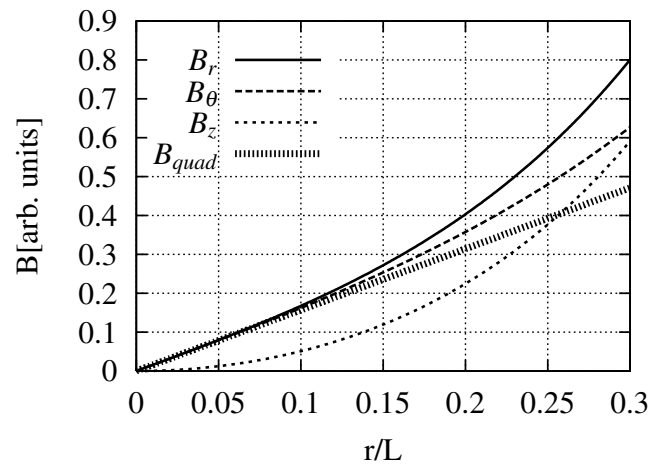

FIG. 1. $\quad r$ dependence of the radial part of both helical and pure quadrupole fields: $B_{r}, B_{\theta}, B_{z}$, and $B_{\text {quad }}$. 
and $\quad B_{z}=\frac{\Phi_{0}}{8}\left((k r)^{2}+\frac{(k r)^{4}}{12}+O\left(r^{6}\right)\right) \cos (2 \theta-k z)$.

For a good linear approximation, the nonlinear terms in the $B_{r}, B_{\theta}$, and $B_{z}$ components have to be negligible compared with the pure quadrupole component. If a $10 \%$ deviation is allowed for either the $B_{r}$ or the $B_{\theta}$ component, the maximum $r$ coordinate $r_{\text {bore }}$ has to keep the following condition:

$$
r_{\text {bore }} \lesssim 0.12 L \text {. }
$$

The same limitation for the $B_{z}$ component is given by

$$
r_{\text {bore }} \lesssim 0.032 L \text {. }
$$

\section{TRANSFER MATRIX OF HELICAL QUADRUPOLE FOCUSING CHANNEL}

A linearized transfer matrix of the HQFC is derived in this chapter. The stability condition for the matrix was deduced by eigenvalue analysis.

\section{A. Cell matrix}

Considering the rotating characteristics of the HQFC's magnetic scalar potential, a transfer matrix from location $s_{a}$ to $s_{b} M_{\mathrm{HQ}}\left(s_{b} \mid s_{a}\right)$ can be written as

$$
M_{\mathrm{HQ}}\left(s_{b} \mid s_{a}\right)=R\left(\frac{k s_{a}}{2}\right) M_{\mathrm{HQ}}\left(s_{b}-s_{a} \mid 0\right) R\left(-\frac{k s_{a}}{2}\right),
$$

where matrix $R$ is a rotation matrix. Using the translation operator Eq. (11), a transfer matrix of the HQFC $M_{\mathrm{HQ}}\left(s_{\text {out }} \mid s_{\text {in }}\right)$ can be divided into the products of small cell matrices:

$$
\begin{aligned}
M_{\mathrm{HQ}}\left(s_{\mathrm{out}} \mid s_{\mathrm{in}}\right) & =\prod_{i=0}^{N-1} M_{\mathrm{HQ}}\left(s_{i+1} \mid s_{i}\right)=\prod_{i=0}^{N-1} R\left(\frac{k s_{i}}{2}\right) M_{\mathrm{HQ}}\left(s_{i+1}-s_{i} \mid 0\right) R\left(-\frac{k s_{i}}{2}\right) \\
& =R\left(\frac{k s_{\mathrm{out}}}{2}\right)\left[\prod_{i=0}^{N-1} R\left(-\frac{k\left(s_{i+1}-s_{i}\right)}{2}\right) M_{\mathrm{HQ}}\left(s_{i+1}-s_{i} \mid 0\right)\right] R\left(-\frac{k s_{\text {in }}}{2}\right),
\end{aligned}
$$

where $\left\{s_{i} \mid i=0, \ldots, N, s_{0}=s_{\mathrm{in}}, s_{N}=s_{\text {out }}\right\}$ is a division of the region $\left[s_{\mathrm{in}}, s_{\text {out }}\right]$. Applying an equal weight division and a limit operator, we obtain

$$
\begin{aligned}
M_{\mathrm{HQ}}\left(s_{\text {out }} \mid s_{\text {in }}\right) & =R\left(\frac{k s_{\text {out }}}{2}\right) \lim _{N \rightarrow \infty}\left[\prod_{i=0}^{N-1} R\left(-\frac{k\left(s_{i+1}-s_{i}\right)}{2}\right) M_{\mathrm{HQ}}\left(s_{i+1}-s_{i} \mid 0\right)\right] R\left(-\frac{k s_{\text {in }}}{2}\right) \\
& =R\left(\frac{k s_{\text {out }}}{2}\right) \lim _{N \rightarrow \infty}\left[\prod_{i=0}^{N-1} R\left(-\frac{k \triangle s}{2}\right) M_{\mathrm{HQ}}(\triangle s \mid 0)\right] R\left(-\frac{k s_{\text {in }}}{2}\right),
\end{aligned}
$$

where $\triangle s$ is the length of slice $\triangle s=\left(s_{\text {out }}-s_{\text {in }}\right) / N$. Considering that a thin helical quadrupole is equivalent to a thin quadrupole under linear approximation, the transfer matrix can be rewritten as

$$
M_{\mathrm{HQ}}\left(s_{\text {out }} \mid s_{\text {in }}\right)=R\left(\frac{k s_{\text {out }}}{2}\right) \lim _{N \rightarrow \infty}\left[\prod_{i=0}^{N-1} R\left(-\frac{k \triangle s}{2}\right) M_{Q}(\triangle s \mid 0)\right] R\left(-\frac{k s_{\text {in }}}{2}\right),
$$

where $M_{Q}(\triangle s \mid 0)$ is a transfer matrix of a thin quadrupole. The matrix $M_{\text {core }}$ is introduced to denote the infinity matrix product in Eq. (14) as follows:

$$
M_{\text {core }}(s)=\lim _{N \rightarrow \infty}\left[\prod_{i=0}^{N-1} R\left(-\frac{k s}{2 N}\right) M_{Q}\left(\frac{s}{N} \mid 0\right)\right]
$$

The infinite matrix product $M_{\text {core }}$ is the product of the cell matrix $R(-k s / 2 N) M_{Q}(s / N \mid 0)$. Because the infinitesimal matrices $R(-k s / 2 N)$ and $M_{Q}(s / N \mid 0)$ become the identity matrix with the limitation $N \rightarrow \infty$, both $(s / N)^{2}$ and higher terms of the infinitesimal matrices are not required to calculate the infinite matrix product. Thus, the higher order terms of $M_{Q}(s / N \mid 0)$ in the eigenvalue problem of the cell matrix are neglected hereafter. The explicit representations of $R(-k s / 2 N)$ and $M_{Q}(s / N \mid 0)$ in

$$
\begin{aligned}
& \text { the transversal phase space }\left(x, x^{\prime}, y, y^{\prime}\right) \text { are given by } \\
& R\left(-\frac{k s}{2 N}\right)=\left(\begin{array}{cccc}
\cos \frac{k s}{2 N} & 0 & \sin \frac{k s}{2 N} & 0 \\
0 & \cos \frac{k s}{2 N} & 0 & \sin \frac{k s}{2 N} \\
-\sin \frac{k s}{2 N} & 0 & \cos \frac{k s}{2 N} & 0 \\
0 & -\sin \frac{k s}{2 N} & 0 & \cos \frac{k s}{2 N}
\end{array}\right)
\end{aligned}
$$

$$
\text { and } M_{Q}\left(\frac{s}{N} \mid 0\right)=\left(\begin{array}{cccc}
1 & \frac{s}{N} & 0 & 0 \\
-K \frac{s}{N} & 1 & 0 & 0 \\
0 & 0 & 1 & \frac{s}{N} \\
0 & 0 & K \frac{s}{N} & 1
\end{array}\right) \text {, }
$$

where $K, q$, and $p$ are the field gradient of the quadrupole field ( $\left.-q k \Phi_{0} / 4 p\right)$, the charge, and the momentum of 
the particle, respectively. Because the sign of the parameter $K$ can be chosen without loss of generality, hereafter we will assume that $K$ is positive.

\section{B. Eigenvalue analysis and stability condition}

The eigenvalue and the eigenvectors of the infinity product in Eq. (15) are derived from those of the cell matrix. The eigenvalue problem of the cell matrix indexed by $s / N$ is defined as

$$
\hat{\lambda}_{i}(s / N) \hat{\mathbf{x}}_{i}(s / N)=R\left(-\frac{k s}{2 N}\right) M_{Q}(s / N \mid 0) \hat{\mathbf{x}}_{i}(s / N) .
$$

Using the eigensolutions of Eq. (18), the eigensolutions of the infinity product $M_{\text {core }}$ can be described as follows:

$$
\mathbf{x}_{i}=\lim _{N \rightarrow \infty} \hat{\mathbf{x}}_{i}(s / N), \quad \lambda_{i}=\lim _{N \rightarrow \infty} \hat{\lambda}_{i}(s / N)^{N} .
$$

After some mathematical operations, we obtained the eigensolution of the matrix $M_{\text {core }}$ as follows:

$$
\left(\lambda_{1}, \lambda_{2}, \lambda_{3}, \lambda_{4}\right)=\left(e^{-i \omega_{H} s}, e^{+i \omega_{H} s}, e^{-i \omega_{L} S}, e^{+i \omega_{L} S}\right),
$$

where $\omega_{H}$ and $\omega_{L}$ are $\sqrt{k^{2} / 4+K}$ and $\sqrt{k^{2} / 4-K}$, respectively. The set of the eigenvectors is

$$
\begin{aligned}
& M_{\text {core }}(s)=\left(\begin{array}{cccc}
C_{p} & \frac{L}{g \pi}\left(g_{p} S_{p}-\frac{S_{m}}{g_{m}}\right) & \frac{S_{m}}{g_{m}} & \frac{L}{g \pi}\left(C_{m}-C_{p}\right) \\
-\frac{\pi}{L} \frac{g}{g_{p}} S_{p} & C_{p} & 0 & \frac{S_{p}}{g_{p}} \\
-\frac{S_{p}}{g_{p}} & \frac{L}{g \pi}\left(C_{p}-C_{m}\right) & C_{m} & \frac{L}{g \pi}\left(\frac{S_{p}}{g_{p}}-g_{m} S_{m}\right) \\
0 & -\frac{S_{m}}{g_{m}} & \frac{\pi}{L} \frac{g}{g_{m}} S_{m} & C_{m}
\end{array}\right) \text {, } \\
& C_{p}=\cos g_{p} \bar{s}, \quad C_{m}=\cos g_{m} \bar{s}, \quad S_{p}=\sin g_{p} \bar{s}, \quad S_{m}=\sin g_{m} \bar{s}, \quad \bar{s}=\frac{\pi s}{L}, \quad g_{p}=\sqrt{1+g}, \\
& g_{m}=\sqrt{1-g} \quad \text { and } \quad g=K\left(\frac{L}{\pi}\right)^{2} .
\end{aligned}
$$

\section{BEAM SIZE IN THE HELICAL QUADRUPOLE FOCUSING CHANNEL}

\section{A. Matched beam boundary}

By definition, the shape of the phase space boundary of a matched beam is conserved after the transfer of one period of a HQFC. Because of the $x-y$ coupling, the analysis has to be performed in a $4 \mathrm{D}$ phase space. In order to build such a boundary, we analyzed the oscillations in the eigenvector space.

Under the stability condition (22), except for the critical conditions $K=k^{2} / 4$ and $K=0$, the whole eigenvalues and eigenvectors were not degenerated. Thus the eigenvalues and the eigenvectors formed two complex conjugate pairs:

$$
\begin{gathered}
\lambda_{1}=\overline{\lambda_{2}}=e^{-i \omega_{H} s}, \quad \mathbf{x}_{1}=\overline{\mathbf{x}_{2}}, \\
\text { and } \quad \lambda_{3}=\overline{\lambda_{4}}=e^{-i \omega_{L} s}, \quad \mathbf{x}_{3}=\overline{\mathbf{x}_{4}} .
\end{gathered}
$$

$$
\begin{aligned}
X & =\left[\mathbf{x}_{1}, \mathbf{x}_{2}, \mathbf{x}_{3}, \mathbf{x}_{4}\right] \\
& =\left(\begin{array}{cccc}
i \frac{2 \omega_{H}}{k} & -i \frac{2 \omega_{H}}{k} & \frac{k}{2 K} & \frac{k}{2 K} \\
\frac{2 K}{k} & \frac{2 K}{k} & 0 & 0 \\
1 & 1 & -i \frac{\omega_{L}}{K} & i \frac{\omega_{L}}{K} \\
0 & 0 & 1 & 1
\end{array}\right),
\end{aligned}
$$

where the vector set $X$ forms the basis of the eigenspace. When both $\omega_{H}$ and $\omega_{L}$ are real, the solutions are periodic and the corresponding motion of a particle is stabilized. Thus the stability condition is given by

$$
0 \leq K \leq \frac{k^{2}}{4}=\frac{\pi^{2}}{L^{2}} \text {. }
$$

The transfer matrix $M_{\mathrm{HQ}}$ can be constructed by $\lambda_{i}$ and $X$ as follows:

$$
\begin{aligned}
M_{\mathrm{HQ}}\left(s_{\text {out }} \mid s_{\text {in }}\right) & =R\left(\frac{k s_{\text {out }}}{2}\right) M_{\text {core }}\left(s_{\text {out }}-s_{\text {in }}\right) R\left(-\frac{k s_{\text {in }}}{2}\right) \\
M_{\text {core }}(s) & =X\left(\begin{array}{cccc}
\lambda_{1} & & 0 & \\
& \lambda_{2} & & \\
0 & \lambda_{3} & \\
0 & & \lambda_{4}
\end{array}\right) X^{-1} .
\end{aligned}
$$

Explicit representation of $M_{\text {core }}(s)$ is given by

where $\overline{\mathbf{x}}$ (overbar) denotes a complex conjugate. In order to represent a physical phase space vector, we used real vectors using linear combination of eigenvectors in each subspace as follows:

$$
\begin{aligned}
\mathbf{x}_{H}\left(A_{H}, \theta_{H}\right) & =A_{H}\left(e^{-i \theta_{H}} \mathbf{x}_{1}+e^{+i \theta_{H}} \overline{\mathbf{x}_{1}}\right), \\
\text { and } \quad \mathbf{x}_{L}\left(A_{L}, \theta_{L}\right) & =A_{L}\left(e^{-i \theta_{L}} \mathbf{x}_{3}+e^{+i \theta_{L}} \overline{\mathbf{x}_{3}}\right),
\end{aligned}
$$

where $A_{H}, A_{L}$ and $\theta_{H}, \theta_{L}$ are the amplitudes and the phases in the subeigenspaces, respectively. Because either set of two parameters $\left(A_{H}, \theta_{H}\right)$ or $\left(A_{L}, \theta_{L}\right)$ covers each whole real subspace, any real vector can be represented by a linear combination of the real vectors: $\mathbf{x}_{H}\left(A_{H}, \theta_{H}\right)$ and $\mathbf{x}_{L}\left(A_{L}, \theta_{L}\right)$. These vectors are transferred by the matrix $M_{\text {core }}(L)$ along one-period length $L$ as follows:

$$
\begin{gathered}
M_{\text {core }}(L) \mathbf{x}_{H}\left(A_{H}, \theta_{H}\right)=\mathbf{x}_{H}\left(A_{H}, \theta_{H}+\omega_{H} L\right), \\
\text { and } M_{\text {core }}(L) \mathbf{x}_{L}\left(A_{L}, \theta_{L}\right)=\mathbf{x}_{L}\left(A_{L}, \theta_{L}+\omega_{L} L\right) .
\end{gathered}
$$


In other words, a transfer of one period corresponds to a rotation in the $2 \mathrm{D}$ subphase space. In a special case, a linear combination of $\mathbf{x}_{H}$ and $\mathbf{x}_{L}$ whose amplitudes $A_{H}$ and $A_{L}$ are constant along the beam axis paints a hyperplane with moving phases $\theta_{H}$ and $\theta_{L}$. According to Eqs. (29) and (30), this particular hyperplane does not change its shape after the transfer of one period.
Because we are considering a 4D phase space, a general beam boundary is described as a hyperplane of the 4D phase space and thus three parameters are required to describe a surface. Selection of the oscillation phases $\theta_{H}$ and $\theta_{L}$ as the parameters is reasonable. As the last parameter, we use an amplitude coupling between two frequency modes that is expressed by trigonometric functions. As a result, one of the matched beam boundaries can be described by following form:

$$
\mathbf{x}_{\text {beam }}(s)=\left\{R\left(\frac{k s}{2}\right)\left[\cos \theta_{0} \mathbf{x}_{H}\left(A_{H}, \omega_{H} s+\theta_{H}\right)+\sin \theta_{0} \mathbf{x}_{L}\left(A_{L}, \omega_{L} s+\theta_{L}\right)\right] \mid \theta_{H}, \theta_{L} \in[0,2 \pi], \theta_{0} \in\left[0, \frac{\pi}{2}\right]\right\},
$$

where $\theta_{H}, \theta_{L}$, and $\theta_{0}$ are the indices of the beam boundary.

\section{B. Emittance and $\sigma$ matrix}

One of the simplest hyperplanes of a matched beam boundary is described by a $4 \times 4 \sigma$ matrix [8] in the following quadratic form:

$$
\begin{gathered}
{\left[\begin{array}{cccc}
x(s) & x^{\prime}(s) & y(s) & y^{\prime}(s)
\end{array}\right][\sigma(s)]^{-1}\left(\begin{array}{c}
x(s) \\
x^{\prime}(s) \\
y(s) \\
y^{\prime}(s)
\end{array}\right)=1,} \\
{[\sigma(s)]^{T}=\sigma(s),}
\end{gathered}
$$

where the superior $T$ denotes the transpose operator. The $\sigma$ matrix that corresponds to the boundary Eq. (31) can be obtained by coordinate transformation from the real phase space to the eigenspace. Considering the relationship between the real phase space and the eigenspace of the matrix $M_{\text {core }}(s)$ in Eq. (23), the coordinate conversion at the point $s$ can be described as

$$
\tilde{\mathbf{v}}(s)=X^{-1} R\left(-\frac{k s}{2}\right) \mathbf{v}(s)
$$

where $\mathbf{v}(s)$ and $\tilde{\mathbf{v}}(s)$ denote a real phase space vector and an eigenspace vector, respectively. Replacing the quadratic form in Eq. (32) with the Hermitian form, the definition of the $\sigma$ matrix is naturally extended to a complex $\tilde{\sigma}$ matrix, which can describe the eigenspace. From Eqs. (32) and (33), $\tilde{\sigma}(s)$ is derived as

$$
\begin{aligned}
{[\tilde{\boldsymbol{\sigma}}(s)]^{-1} } & =X^{*} R\left(\frac{k s}{2}\right)^{*}[\sigma(s)]^{-1} R\left(\frac{k s}{2}\right) X \Leftrightarrow \sigma(s) \\
& =R\left(\frac{k s}{2}\right) X \tilde{\boldsymbol{\sigma}}(s) X^{*} R\left(-\frac{k s}{2}\right),
\end{aligned}
$$

where $*$ is the Hermitian operator. Thus we can obtain the $\sigma$ matrix from the Hermitian form of the hyperplane in the eigenspace. Because of the trigonometric functions in Eq. (31), we can easily write down the Hermitian form as follows:

$$
\begin{aligned}
& \tilde{\mathbf{v}}(s)=\left(\begin{array}{c}
A_{H} e^{-i\left(\theta_{H}+\omega_{H} s\right)} \cos \theta_{0} \\
A_{H} e^{+i\left(\theta_{H}+\omega_{H} s\right)} \cos \theta_{0} \\
A_{L} e^{-i\left(\theta_{L}+\omega_{L} s\right)} \sin \theta_{0} \\
A_{L} e^{+i\left(\theta_{L}+\omega_{L} s\right)} \sin \theta_{0}
\end{array}\right), \\
& {[\tilde{\mathbf{v}}(s)]^{*}\left(\begin{array}{cccc}
\frac{1}{2 A_{H}^{2}} & & & \\
& \frac{1}{2 A_{H}^{2}} & & \\
& & \frac{1}{2 A_{L}^{2}} & \\
\mathbf{0} & & \frac{1}{2 A_{L}^{2}}
\end{array}\right) \tilde{\mathbf{v}}(s)=1 .}
\end{aligned}
$$

Then the explicit form of $\sigma(s)$ can be obtained as

$$
\begin{aligned}
\sigma(s) & =R\left(\frac{k s}{2}\right) X\left(\begin{array}{cccc}
2 A_{H}^{2} & & 0 & \\
& 2 A_{H}^{2} & & \\
& & 2 A_{L}^{2} & \\
0 & 2 A_{L}^{2}
\end{array}\right) X^{*} R\left(-\frac{k s}{2}\right) \\
& =R\left(\frac{k s}{2}\right)\left(\begin{array}{cccc}
4 \frac{k^{2}+4 K}{k^{2}} A_{H}^{2}+\frac{k^{2}}{K^{2}} A_{L}^{2} & 0 & 0 & 2 \frac{k}{K} A_{L}^{2} \\
0 & 16 \frac{K^{2}}{k^{2}} A_{H}^{2} & 8 \frac{K}{k} A_{H}^{2} & 0 \\
0 & 8 \frac{K}{k} A_{H}^{2} & 4 A_{H}^{2}+\frac{k^{2}-4 K}{K^{2}} A_{L}^{2} & 0 \\
2 \frac{k}{K} A_{L}^{2} & 0 & 0 & 4 A_{L}^{2}
\end{array}\right) R\left(-\frac{k s}{2}\right) .
\end{aligned}
$$

From the relationship among the Twiss parameters, the emittance and $2 \times 2 \sigma$ submatrices, the following relationships were derived:

$$
\sigma(s)=\left(\begin{array}{ll}
\sigma_{x x}(s) & \sigma_{x y}(s) \\
\sigma_{y x}(s) & \sigma_{y y}(s)
\end{array}\right)
$$




$$
\begin{aligned}
\mathcal{E}=\sqrt{\operatorname{det} \sigma(s)}, & \varepsilon_{x}(s)=\sqrt{\operatorname{det} \sigma_{x x}(s),} \\
\varepsilon_{y}(s)= & \sqrt{\operatorname{det} \sigma_{y y}(s),}
\end{aligned}
$$

where $\mathcal{E}, \varepsilon_{x}$, and $\varepsilon_{y}$ are $4 \mathrm{D}$ emittance, $x$ - $x^{\prime}$ subphase space emittance, and $y-y^{\prime}$ subphase space emittance, respectively. The $4 \mathrm{D}$ emittance $\mathcal{E}$ is a constant, because of Liouville's theorem. In a decoupled case, $\varepsilon_{x}(s)$ and $\varepsilon_{y}(s)$ become constants and $4 \mathrm{D}$ emittance $\mathcal{E}$ is reduced to $\varepsilon_{x} \varepsilon_{y}$.

For a comparison with FODO focusing system, we considered the symmetric emittance for $x$ and $y$ directions, and thus the ratio $A_{L} / A_{H}$ was deduced from the condition $\varepsilon_{x}(s)=\varepsilon_{y}(s)$,

$$
\frac{A_{L}}{A_{H}}=\frac{2 K}{k}\left(\frac{k^{2}+4 K}{k^{2}-4 K}\right)^{1 / 4} .
$$

Fortunately the dependence of $s$ does not appear in this constraint condition.

In order to compare the beam size with the conventional FODO lattice, we introduced the normalized envelope functions $\hat{\beta}$ and $\hat{\gamma}$. These functions were defined so as to become the ordinary Twiss parameters when the phase spaces $x$ and $y$ were decoupled. By calculating the envelope of Eq. (31) with the condition Eq. (39), the normalized envelope functions can be obtained as follows:

$$
\begin{aligned}
& \hat{\beta}_{x}(s)=\frac{\left[x_{\mathrm{env}}(s)\right]^{2}}{\sqrt{\mathcal{E}}} \\
&=L \frac{\pi}{G}\left(\frac{1+\frac{G}{\pi^{2}} \cos ^{2} \frac{k s}{2}}{\sqrt{1+\frac{G}{\pi^{2}}}}+\frac{1-\frac{G}{\pi^{2}} \sin ^{2} \frac{k s}{2}}{\sqrt{1-\frac{G}{\pi^{2}}}}\right), \\
& \hat{\gamma}_{x}(s)=\frac{\left[x_{\mathrm{env}}^{\prime}(s)\right]^{2}}{\sqrt{\mathcal{E}}}=\frac{1}{L} \frac{G}{\pi}\left(\frac{\cos ^{2 \frac{k s}{2}}}{\sqrt{1+\frac{G}{\pi^{2}}}}+\frac{\sin ^{2} \frac{k s}{2}}{\sqrt{1-\frac{G}{\pi^{2}}}}\right), \\
& \hat{\beta}_{y}(s)=\hat{\boldsymbol{\beta}}_{x}\left(s+\frac{L}{2}\right) \text { and } \hat{\gamma}_{y}(s)=\hat{\gamma}_{x}\left(s+\frac{L}{2}\right),
\end{aligned}
$$

where $G$ is the dimensionless parameter $K L^{2}$.

And thus, the index of the maximum beam size $\Gamma_{+}=$ $\hat{\beta}_{\max } / L$ can be described as

$$
\Gamma_{+}(G)=\frac{\pi}{G}\left(\sqrt{1+\frac{G}{\pi^{2}}}+\frac{1}{\sqrt{1-\frac{G}{\pi^{2}}}}\right)
$$

The linear approximation condition Eqs. (9) and (10) can be rewritten by $\Gamma_{+}$as follows:

$$
\text { Eq. (9) } \rightarrow \frac{\sqrt{\mathcal{E}}}{L} \lesssim \frac{0.014}{\Gamma_{+}},
$$

$$
\text { Eq. }(10) \rightarrow \frac{\sqrt{\mathcal{E}}}{L} \lesssim \frac{0.0010}{\Gamma_{+}} \text {. }
$$

\section{COMPARISON WITH FODO}

\section{A. Linear approximation}

In order to compare a HQFC with the FODO lattice that has four equal length cells (each cell length is $\frac{L}{4}$ ), the $\Gamma_{+}$ of the FODO lattice has to be considered. For a symmetrical FODO, beta functions of $x-x^{\prime}$ and $y-y^{\prime}$ planes are described by the same functions with a phase displacement. Using the one-period transfer matrix $M_{\text {FODO }}$ starting from the center of focusing cell, the maximum value of the beta function in $x-x^{\prime}$ plane $\beta_{x \max }$ can be obtained as

$$
\Gamma_{+}=\frac{\beta_{x \max }}{L}=\frac{\left|M_{\mathrm{FODO} x x^{\prime}}\right|}{L \sqrt{1-\left(\frac{\mathrm{tr} M_{\mathrm{FODO}}}{2}\right)^{2}}} .
$$

The focusing power of a HQFC is twice as large as that of a FODO lattice with same $K$, because a HQFC can be considered as a superposition of a conventional FODO and a skewed FODO with longitudinal displacement. We did not consider a FFDD lattice (a variation of FODO lattice replacing its drift spaces with the focusing and defocusing elements) having a magnet population the same as a HQFC, because the $\Gamma_{+}$value does not have a significant improvement from a FODO lattice. Figure 2 shows the $G$ dependences of the normalized maximum beam size $\sqrt{\Gamma_{+}}$for HQFC and FODO. The beam size curve of a HQFC looks like a half-scaled curve of a FODO. It is explained by the fact that a HQFC is complex of a FODO cell and a skewed FODO cell, but the minimum beam size $\sqrt{\Gamma_{+ \text {min }}}$ is different between two structures. The minimum beam size of a HQFC is $5 \%$ smaller than FODO's minimum beam size. The difference of the minimum beam size between a HQFC and a FODO is small, but the scale difference of $G$ parameter makes a big advantage to fabricate a transport channel for higher momentum particles by HQFC structure with realistic constraint.

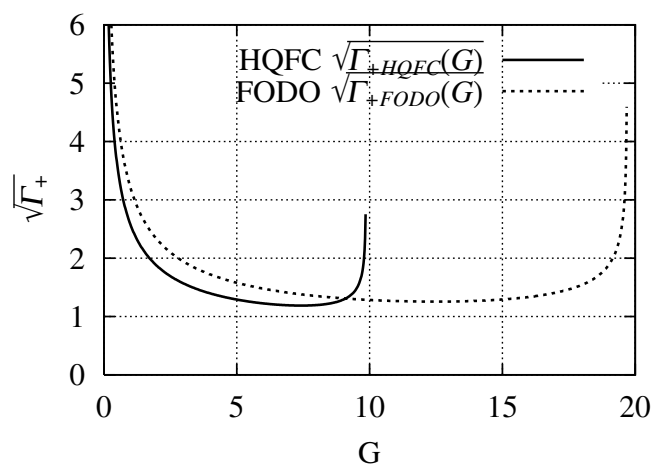

FIG. 2. Comparison of $\sqrt{\Gamma_{+}}$between HQFC and FODO. 
Considering that the maximum magnetic flux density in iron limits the strength of a quadrupole magnet, the magnetic flux density on the pole surface $B_{\text {pole }}$ is a useful index for comparison of magnets. Under the linear approximation, the relationship between the emittance $\varepsilon=$ $\sqrt{\mathcal{E}}$ and the maximum magnetic flux density on the pole surface $B_{\text {pole }}$ can be written as

$$
\begin{aligned}
B_{\text {pole }} & \sim \frac{r_{\text {bore }}}{r_{\text {beam }}} \frac{d B_{y}}{d x} x_{\text {max }}=\frac{r_{\text {bore }}}{r_{\text {beam }}} K B \rho \sqrt{\Gamma_{+} L \varepsilon} \\
& =\frac{r_{\text {bore }}}{r_{\text {beam }}} \frac{G}{L^{\frac{3}{2}}} B \rho \sqrt{\Gamma_{+} \varepsilon},
\end{aligned}
$$

where $B \rho$ is the momentum of the particle. $r_{\text {beam }} / r_{\text {bore }}$ is a relative margin of a beam channel, which is usually less than 1 because of the vacuum beam pipe. Comparing a $\mathrm{HQFC}$ and a FODO lattice that have the same relative margins and acceptances, the $B_{\text {pole }}$ of a HQFC is only $30 \%$ of that of a FODO. This significantly decreases the excitation loss.

\section{B. Nonlinear case}

Beyond the linear region, the effect of nonlinear terms must be included. In order to evaluate the nonlinear effect, we tracked particles numerically in the nonlinear magnetic field described by Eqs. (3)-(5).

Figure 3 shows the beam size including the nonlinear effect that is normalized by the initial emittance around the condition Eqs. (44) and (45). These normalized beam sizes were obtained from the particle tracking simulations whose initial distribution was the paraxial approximated matched beam ellipse. At some points in Fig. 3, the normalized beam sizes blow up with the increase of the initial beam size. This instability, which causes such a blowing up, comes from the effect of the nonlinear field at the perimeter. This instability appears from the low momentum region (high $G$ region), because of excessive focusing power. Because the particles beyond the instability region were lost from the channel bore, the normalized beam sizes are not shown.

In order to examine the relationship between the stable region width and the linear approximation condition, the

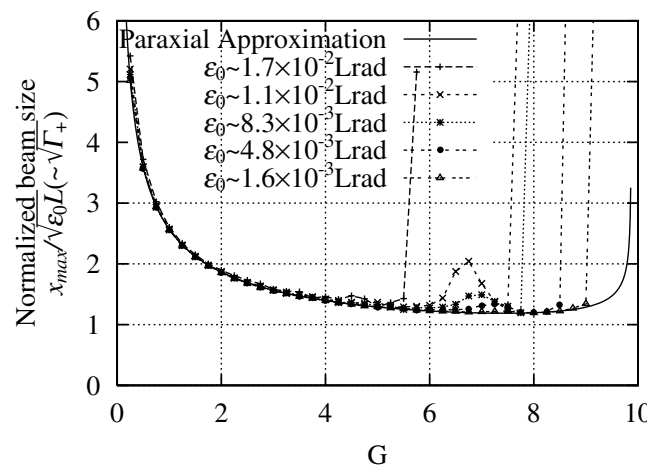

FIG. 3. Normalized beam size calculated by particle tracking.

parameter $\Gamma_{+} \sqrt{\mathcal{E}} / L$ at the higher edge of the stable $G$ region is compared with Eqs. (44) and (45). Considering that the initial emittance $\varepsilon_{0}$ equals to $\sqrt{\mathcal{E}}$ of Eqs. (44) and (45), the critical parameters $\Gamma_{+} \sqrt{\mathcal{E}} / L$ of the initial emittances $\varepsilon_{0} / L \sim 1.7 \times 10^{-2}, \quad 8.3 \times 10^{-3}, \quad$ and $1.6 \times$ $10^{-3} \mathrm{rad}$ are $2.7 \times 10^{-2}, 1.2 \times 10^{-2}$, and $2.7 \times$ $10^{-3} \mathrm{rad}$, respectively. The parameters $\Gamma_{+} \sqrt{\mathcal{E}} / L$ obtained from Fig. 3 are not constant and the width of the parameter $\Gamma_{+}$related to a change of 10 times of the parameters $\Gamma_{+} \sqrt{\mathcal{E}} / L$ is about $\pm 10 \%$. Therefore, the width of the stable region is not directly determined by the relative strength of the nonlinear field. We have to look up the stable region chart as in Fig. 3 in designing a HQFC.

\section{DISCUSSION AND CONCLUSION}

Considering the above results and the existence of the $x-y$ coupling, a HQFC is suitable for transportation of high momentum secondary particles by a small aperture channel. The beam transport channels with $100 \mathrm{~mm}$ radius for the $300 \mathrm{MeV}$ and $1 \mathrm{GeV}(B \rho \sim 1.3,3.7 \mathrm{~T} \mathrm{~m})$ muon beams with $\pm 50 \%$ momentum spread can be considered as one example. Table I shows the parameters derived from an assumption of the matched beam and results shown in Fig. 3, where the magnetic flux density at the pole tip is limited up to $1.4 \mathrm{~T}$. The nonlinear effects of the magnetic field of a HQFC are taken into account in Table I.

TABLE I. Example of HQFC and FODO.

\begin{tabular}{lccccc}
\hline \hline Muon condition & Energy $[\mathrm{MeV}]$ & & 300 & & 1000 \\
& Momentum $\left[\mathrm{MeV} / \mathrm{c}^{2}\right]$ & & 390 & \multicolumn{2}{c}{1109} \\
& $B \rho[\mathrm{Tm}]$ & & 1.3 & & 3.7 \\
Channel parameter & Field type & FODO & HQFC & FODO & HQFC \\
& Field period $L[\mathrm{~m}]$ & 1.02 & 0.724 & 1.71 & 1.18 \\
Field parameter at center momentum & $G=K L^{2}$ & 11.1 & 5.00 & 11.1 & 5.00 \\
& Field gradient $[\mathrm{T} / \mathrm{m}]$ & 14.0 & 12.4 & 14.0 & 13.4 \\
Acceptance at $p_{\text {center }}$ & $\varepsilon_{\text {center }}[\mathrm{m} \mathrm{rad}]$ & $6.16 \times 10^{-3}$ & $8.29 \times 10^{-3}$ & $3.65 \times 10^{-3}$ & $5.10 \times 10^{-3}$ \\
Minimum acceptance at edge of momentum spread & $\varepsilon_{\min }[\mathrm{m} \mathrm{rad}]$ & $5.16 \times 10^{-3}$ & $6.14 \times 10^{-3}$ & $3.06 \times 10^{-3}$ & $3.80 \times 10^{-3}$ \\
& $\varepsilon / \mathrm{L}[\mathrm{rad}]$ & $5.09 \times 10^{-3}$ & $8.49 \times 10^{-3}$ & $1.78 \times 10^{-3}$ & $3.21 \times 10^{-3}$ \\
\hline \hline
\end{tabular}


The bore radius and the maximum magnetic field assumed in the example can be achieved by conventional room temperature magnet technology. While a helical quadrupole with an electromagnet may have difficulties in the fabrication of helical poles and the coil assembly, one with permanent magnets should be feasible.

As the result of nonlinear effect of Bessel function term, the field gradient of HQFC is smaller than FODO's. But, a HQFC has about $20 \%$ larger acceptances than a FODO channel in two cases. For a given acceptance, a smaller bore radius is sufficient for a HQFC.

[1] F. S. Chute, F. E. Vermeulen, and A. Youssef, Nucl. Instrum. Methods 82, 86 (1970).
[2] E. A. Youssef, F.S. Chute, and F. E. Vermeulen, Nucl. Instrum. Methods 93, 181 (1971).

[3] R. G. McPherson, F. E. Vermeulen, and F. S. Chute, Nucl. Instrum. Methods 148, 5 (1978).

[4] Y. Mori et al., in Proceedings of the 8th Symposium on Accelerator Science and Technology, Saitama, Japan (Ionics Publishing Company, Ltd., Tokyo, 1991), pp. 182-184.

[5] L. Xiu, S. Ohnuma, and W. Wang, in Proceedings of the 1993 Particle Accelerator Conference, Washington, D.C. (IEEE, Piscataway, NJ, 1993), pp. 3148-3150.

[6] H. O. Funsten, Nucl. Instrum. Methods 44, 301 (1966).

[7] K. J. LeCouteur, Plasma Phys. 9, 457 (1967).

[8] K. R. Crandall, TRACE 3-D Documentation, Los Alamos National Laboratory Report No. LA-UR-97886, 1987. 